\title{
Utility of Ipsilateral Medial Fibular Transport Using the Ilizarov Frame in the Treatment for Non-elderly Patients Sustaining Massive Tibial Bone Defects as a Sequela of Trauma and Infection: A Systematic Review
}

\author{
Patricio E. Dumlao III, MD and Gracia Cielo E. Balce, MD \\ Department of Orthopedics, Philippine General Hospital, University of the Philippines Manila
}

\begin{abstract}
Introduction and Objectives. Segmental tibial bone loss from tumor, trauma, or infection is a debilitating, limbthreatening scenario where treatment principles involve aggressive resection of infected tissues usually leading to large defects requiring reconstruction. A systematic review was conducted to determine the best available evidence related to the ipsilateral medial fibular transport using the llizarov frame in the management of these massive tibial bone defects.
\end{abstract}

Methods. Multiple medical online database search for articles containing the keywords: ipsilateral medial fibular transport, medial fibula transport, medialization of the fibula using the llizarov fixator, ring external fixator, vascularized free fibula, vascularized fibula transfer, and other related MeSH terms was done. Data was summarized to describe the mean age, bone defect, external fixator time, external fixator index, and bone and functional results using the ASAMI criteria.

Results. Eight studies with a total of 43 patients with massive tibial bone defects treated by fibular transport using the llizarov methods were identified. The mean age was 25.27 years (6.5-44.4) with a mean bone defect of 13.57 $\mathrm{cm}$ (9.52-17). The mean length of follow-up was 37.67 months (18-70.2). The bone union rate was $100 \%$. Mean external fixation time was 9.59 months (8.31-10.88) and external fixation index was 0.61 months/cm (0.52-0.70). The majority of patients have an excellent bone (84\%) and functional (52\%) results. The average rate of complication was determined at $0.74 /$ patient $(95 \% \mathrm{Cl}, 0.60-0.89)$. The most common complications include pin-tract infection (37\%), residual loss of motion/stiffness of knee and ankle (35\%), and pain on the transport site (21\%).

Conclusion. Ipsilateral medial fibular transport using the llizarov frame provides a viable alternative treatment option for the treatment of massive tibial bone defects.

Key Words: Tibial bone defect (S82), llizarov technique (E04), External fixation devices (E07)

\section{INTRODUCTION}

Finalist - Presented in the $69^{\text {th }}$ Annual Convention Philippine Orthopedics Association: Annual Residents' Research Forum on November 2018, at the EDSA Shangri-La Hotel, Mandaluyong City.

Corresponding author: Patricio E. Dumlao III, MD

Department of Orthopedics

Philippine General Hospital

University of the Philippines Manila

Email: patricio3dumlao@rocketmail.com
Segmental tibial bone loss from tumor, trauma, or infection is a debilitating, limb-threatening scenario that poses a challenge for reconstructive surgery. ${ }^{1}$ 1) For tumor or neoplasms, the long bone diaphysis is the predilection site of a multitude of benign and malignant conditions necessitating wide resection and consequently result in bone defects requiring reconstruction. ${ }^{2}$ 2) High velocity lower extremity trauma resulting in mangled extremities and its degree of soft tissue injury and contamination coupled with severe bone comminution could result in massive bone loss. ${ }^{3}$ 
Ipsilateral Medial Fibular Transport in the Treatment of Massive Tibial Bone Defects

3) Osteomyelitis is a severe bone infection from an array of etiologies. The cornerstone of treatment is to remove the sequestrum and other devitalized tissue that harbor bacteria. Radical resection of bone leads to segmental defects. ${ }^{4}$

The common causes infrequently occur as a combination such as that of trauma with resultant infection. Open fracture of the tibia has a reported incidence of developing posttraumatic osteomyelitis from $4.5-20 \%$ directly correlated with the severity of the injury. ${ }^{5}$ Reported failure rate of $30 \%$ for the initial fracture fixation and soft-tissue coverage was identified among patients who developed posttraumatic tibial osteomyelitis. Treatment principles at this stage involve aggressive resection of infected tissues usually leading to large defects requiring reconstruction. ${ }^{6}$

The mainstay of success for surgical intervention is adequate dead space management whether soft tissue or osseous defects because of the radical debridement. Management of the reconstruction of bony defects have involved several methods, such as healing by secondary intention, closed irrigation systems, antibiotic polymethylmethacrylate (PMMA) beads, iliac crest bone graft, autologous bone graft, and vascularized fibular graft. ${ }^{7}$ Healing by secondary and intention and closed irrigation systems were deemed lacking the capacity to resolve the infection. PMMA beads and spacers were only a temporizing measure for stabilization. ${ }^{7}$ Bone loss of $>6 \mathrm{cms}$ and/or $>30 \%$ volume defect require reconstruction with bone transfer and other methods with an adjunct stabilizing frame. Vascularized fibular bone has been described as the treatment of large bone defects and is considered most appropriate for reconstruction owing to its anatomic geometry, length, strength, vascular pedicle, and capability for hypertrophy, with a success rate of as high as $80 \%$ but these procedures are complex. ${ }^{8,9}$ Presenting as an alternate volition to vascularized fibular grafts involve the bone transport methods hailing from Ilizarov techniques. The classical method owes its success to the principle of distraction osteogenesis in that the new bone formed is highly vascular. Also, the capability of the Ilizarov frame for both distraction and compression of an osteotomy and docking site respectively offers a versatile method of reconstruction for several host types. ${ }^{5}$ Hosts include those with $>6 \mathrm{cms}$ osseous defect with varying loss of soft tissue due to surgical eradication of nonviable, infected, or contaminated components. ${ }^{5}$ The use of the Ilizarov frame in addressing the stability and reconstructive issues has been described to achieve a high success rate (90\%) in treating osteomyelitis. As an offshoot of the versatility of the Ilizarov frame, the capability for transverse bone transport has been underutilized. The concept of ipsilateral medial fibular transport using the Ilizarov frame is a novel construct in the management of large tibial defects. ${ }^{10}$ The adaptability of the frame for incremental transport of the fibula to an adjacent defect and subsequent compression upon alignment on docking sites provides less soft tissue violation in the minimal surgical dissection required. ${ }^{11}$ Introduced only in 1998 and since then have only been recorded in case reports and series, in addition to the heterogeneity of patient population and indication for the treatment option, studies have yet to be summarized to identify the utility and outcomes of such a procedure. ${ }^{10,11}$ A systematic review was then conducted to determine the best available evidence related to the ipsilateral medial fibular transport using the Ilizarov frame in the management of massive tibial bone defects.

\section{METHODS}

\section{Criteria for inclusion studies in this review}

Type of studies: ideally randomized controlled trials, but in the absence of randomized evidence, non-randomized studies were considered i.e. cohort, case-control, case series

Types of participants: patients sustaining massive tibial bone defects from both trauma and infection, treated primarily with ipsilateral medial fibular transport using the Ilizarov frame

Types of outcome measures: bone transport time, external fixation time, total treatment time, bone results, functional outcome, and complications

\section{Data source}

Articles were searched using the keywords: ipsilateral medial fibular transport, medial fibula transport, medialization of the fibula using the Ilizarov fixator, ring external fixator, vascularized free fibula, vascularized fibula transfer, and other related $\mathrm{MeSH}$ terms.

Databases searched: Medline, Pubmed, Cochrane Central Register of Controlled Trials (CENTRAL), International Network for the Availability of Scientific Publications (INASP), Google Scholar, Up to Date.

\section{Data analysis}

Studies gathered were appraised for directness and validity of the respective research question, effect of treatment (i.e., efficacy of treatment as described by the measured outcomes), and applicability to the patient population. The limitations and possible sources of bias were identified. The Cochrane manual for appraisal and synthesis of randomized and non-randomized studies was used as a guide. Descriptive statistics using weighted means and tests for heterogeneity were done to characterize the study populations. The interventions and outcomes were compared to recent evidence based on the appropriate applicable test for significance.

\section{RESULTS}

The preliminary literature search recognized 1250 records from databases and other sources matching the keywords, respectively. The search was refined down to 68 pertinent records circulated from January 1997 to November 2017. Twenty-three (23) studies remained after selection by perusing titles, headings, and abstracts. Ultimately, 8 studies 


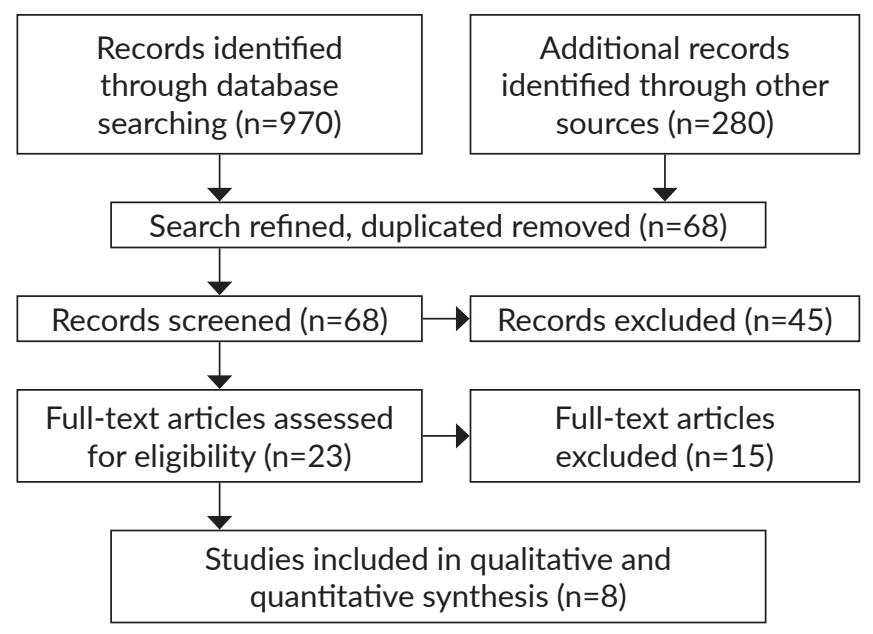

Figure 1. Flow chart showing the quantity of studies assessed at each phase in the systematic review.

fulfilled the inclusion criteria in the systematic review by reading the full-text articles. (Figure 1)

Among the involved studies, 6 were retrospective case series, ${ }^{10,12-18}$ and 2 were case reports. ${ }^{13,19}$ The systematic review included a total of 43 patients with massive tibial defects treated by fibular transport using the Ilizarov methods.
The mean age of all patients was 25.27 years (6.5-44.4); the mean bone loss/defect was $13.57 \mathrm{~cm}(9.52-17)$. The mean length of follow-up was 37.67 months (18-70.2). Additional information was listed in Table 1.

The standard treatment included radical debridement and resection, antibiotic treatment, and Ilizarov concepts and procedures. Ilizarov techniques included bone transport (both ipsilateral tibia and fibula corticotomy), acute compression and lengthening, and docking. All included studies reported bony union. The mean external fixation time (EFT) was 9.59 months (8.31-10.88) and the mean external fixation index (EFI) was 0.61 months/cm (0.52-0.70) (Table 2).

The criteria recommended by the Association for the Study of the Method of Ilizarov (ASAMI) were applied to appraise bone and functional results in the studies. Bone results utilized the following 4 parameters: 1) union, 2) infection, 3) deformity and 4) limb-length discrepancy. Functional results whereas made use of the following 5 considerations for evaluation: 1) level of activity (active, inactive, amputation),2) limp,3) stiffness (knee or ankle joint), 4) reflex sympathetic dystrophy, and 5) pain. Heterogeneity of the population was determined, and effect size (fixed and random effects) analysis presented the weighted means (frequency) of excellent, good, fair, and poor rate in bone and functional results as listed in Table 2.

Table 1. Studies included and general characteristics

\begin{tabular}{|c|c|c|c|c|c|c|c|c|c|c|}
\hline Author & $\begin{array}{l}\text { Study } \\
\text { No. }\end{array}$ & Year & $\begin{array}{l}\text { Study } \\
\text { design }\end{array}$ & $\begin{array}{l}\text { Number of } \\
\text { patients }\end{array}$ & $\begin{array}{l}{ }^{*} \text { due to } \\
\text { trauma }\end{array}$ & $\begin{array}{l}\text { *due to } \\
\text { infection }\end{array}$ & $\begin{array}{l}\text { *due to } \\
\text { tumor }\end{array}$ & $\begin{array}{l}\text { Mean age } \\
\text { (years) }\end{array}$ & $\begin{array}{l}\text { Mean tibial bone } \\
\text { defect }(\mathrm{cm})\end{array}$ & $\begin{array}{l}\text { Follow-up } \\
\text { (months) }\end{array}$ \\
\hline Zaman A., et al. & 1 & 2017 & RCS & 12 & 0 & 12 & 0 & 8.25 & 9.52 & 24.0 \\
\hline Al-Sayyad M., et al. & 2 & 2015 & RCS & 6 & 2 & 4 & 0 & 8.00 & 9.80 & 24.0 \\
\hline Yin P., et al. & 3 & 2015 & RCS & 9 & 8 & 0 & 1 & 32.22 & 15.60 & 40.0 \\
\hline Shafi R., et al. & 4 & 2008 & $\mathrm{CR}$ & 1 & 1 & 0 & 0 & 42.00 & 15.00 & 18.0 \\
\hline Shiha A., et al. & 5 & 2008 & RCS & 2 & 1 & 1 & 0 & 6.50 & 11.50 & 36.0 \\
\hline Catagni M., et al. & 6 & 2006 & RCS & 7 & 4 & 3 & 0 & 40.86 & 15.40 & 70.0 \\
\hline Atkins R., et al. & 7 & 1999 & $\mathrm{RCS}$ & 5 & 3 & 2 & 0 & 44.40 & 14.75 & 70.2 \\
\hline Kim H., et al. & 8 & 1998 & $\mathrm{CR}$ & 1 & 1 & 0 & 0 & 20.00 & 17.00 & 19.2 \\
\hline
\end{tabular}

${ }^{*} R C S=$ retrospective case series, $C R=$ case report

Table 2. Outcomes of interventions

\begin{tabular}{|c|c|c|c|c|c|c|}
\hline Study no. & Union no. & $\begin{array}{c}\text { Bone results - } \\
\text { ASAMI (E/GF/P) }\end{array}$ & $\begin{array}{c}\text { Functional results - } \\
\text { ASAMI (E/G/F/P) }\end{array}$ & Comp. (per patient) & EFT (months) & EFI (months/cm) \\
\hline 1 & $12(100 \%)$ & $12 / 0 / 0 / 0$ & $0 / 12 / 0 / 0$ & 0.60 & 5.80 & 0.29 \\
\hline 2 & $6(100 \%)$ & $5 / 1 / 0 / 0$ & $5 / 1 / 0 / 0$ & 1.00 & 11.08 & 1.12 \\
\hline 3 & 9 (100\%) & $9 / 0 / 0 / 0$ & $6 / 3 / 0 / 0$ & 1.67 & 11.67 & 0.75 \\
\hline 4 & $1(100 \%)$ & $1 / 0 / 0 / 0$ & $1 / 0 / 0 / 0$ & 1.00 & 13.40 & 0.89 \\
\hline 5 & 2 (100\%) & $1 / 1 / 0 / 0$ & $1 / 0 / 0 / 0$ & 1.00 & 2.75 & 0.24 \\
\hline 6 & 7 (100\%) & $4 / 3 / 0 / 0$ & $1 / 6 / 0 / 0$ & 0.28 & 11.14 & 0.72 \\
\hline 7 & 5 (100\%) & $4 / 1 / 0 / 0$ & $0 / 4 / 1 / 0$ & 0.40 & 4.90 & 0.30 \\
\hline 8 & $1(100 \%)$ & $1 / 0 / 0 / 0$ & $1 / 0 / 0 / 0$ & 0.00 & 16.00 & 0.59 \\
\hline
\end{tabular}

${ }^{*}$ ASAMI $=$ Association for the Study of the Method of llizarov, E/G/F/P $=$ Excellent/Good/Fair/Poor, EFT = external fixation time, EFI $=$ external fixation index 
Ipsilateral Medial Fibular Transport in the Treatment of Massive Tibial Bone Defects

Table 3. Complications

\begin{tabular}{clcc}
\hline Study & \multicolumn{1}{c}{ Complications } & Population & Outcome \\
\hline 1 & 7 (3 pintract infection, 4 residual loss of motion, ${ }^{* *} 2$ fracture) & 12 & 0.60 \\
2 & 6 (4 pintract infection, 2 residual loss of motion) & 6 & 1.00 \\
3 & 15 (6 pintract infection, 9 residual loss of motion) & 9 & 1.67 \\
4 & 1 pintract infection & 1 & 1.00 \\
5 & 2 (1 pintract infection, ${ }^{*}$ 1 limb-length discrepancy) & 2 & 1.00 \\
6 & 2 pintract infection & 7 & 0.28 \\
7 & 2 (2 pintract infection, ${ }^{*} 5$ residual loss of motion, ${ }^{* * *} 1$ amputation) & 5 & 0.40 \\
8 & 0 (*1 limb-length discrepancy) & 1 & 0.00 \\
\hline
\end{tabular}

${ }^{*}=$ not significant to affect outcome (ASAMI), ${ }^{* *}=$ not on the transport site,${ }^{* * *}=$ neoplasm necessitating amputation occurred five months upon removal of fixator

The average rate of complication was determined at 0.74/patient (95\% CI, 0.60-0.89). The most common complications include pin-tract infection (37\%), residual loss of motion/stiffness of knee and ankle (35\%), and pain on the transport site (21\%). Other complications are listed in Table 3.

\section{DISCUSSION}

The systematic review included 8 studies, and descriptive statistics were done in the analysis to evaluate the efficacy of the ipsilateral fibular transport using Ilizarov methods in the management of massive tibial bone defects.

The rate of incidence for excellent bone results was $84 \%$ with a corresponding effect size of 0.82 (95\% CI, 0.59-1.09) and excellent functional results were determined at $52 \%$ with an effect size of 1.25 (95\% CI, 08.3-1.67). The data with their computed $Q$ value showed that the values were not statistically heterogeneous. Thus, the outcomes presented that most patients treated with the ipsilateral fibular transport using the Ilizarov method showcased excellent bone and functional results.

The systematic analysis presented a mean EFT of 9.59 months (95\% CI, 8.31-10.88). The EFT compared to recent studies on the treatment of post-traumatic defects, and infected non-union showed that more than $80 \%(z=0.31$ (post-traumatic), $\mathrm{z}=0.47$ (infected nonunion)) of patients treated with the non-fibular transport configuration had longer EFTs. ${ }^{20,21}$ Also the computed mean for the EFI was at 0.61 months/cm (95\% CI, 0.53-0.70) when compared to the previous reference studies exhibited $65.87 \%(\mathrm{z}=1.00$ for both post-traumatic and infected nonunion) of patients treated with the conventional bone transport had a larger-longer EFI. This is consistent with a more efficient transport concerning the length of defect bridged about time on the fixator for the fibular transport. Compared to a more recent study in the use of bone transport in lower limb reconstruction, the EFT of their study was calculated at 4.05 months $(95 \%$ CI, 0.74$4.79, \mathrm{P}=0.01$ ), significantly shorter than our value. ${ }^{22}$ Though, their EFI calculated at 9.16 months/cm (95\% CI, 1.71-10.86, $\left.\mathrm{P}=9.17 \mathrm{X} 10^{-10}\right)$ is statistically longer than our study.
The population exhibited heterogeneity in reported complications (Q value $=10.65$ (fixed) and 6.67 (random), $\mathrm{df}=7$ ). The differences in the reported complications are attributed to the lack of standardized reporting, diverse research quality, different surgeons' experience, and multiplicity of post-operative protocols. ${ }^{21}$ Pin-site infection ranked as the most frequently reported complication with an incidence of $37 \%$ among all patients. It is important to note that the reported rate is lower than the $47.8 \%$ average occurrence of pin-tract infection in patients using circular fixators for greater than 6 months as reported in a systematic review..$^{22}$ In conjunction, all pin-tract infections responded to local tract care and none necessitated surgical intervention. Pain on the fibular transport site was reported in $21 \%$ of cases which resolved through oral pain medications. No patient needed the use of narcotics. ${ }^{17}$ Two studies described residual limb length discrepancy of less than $2.5 \mathrm{~cm}$ deemed as a non-complication using the ASAMI criteria. ${ }^{16,19}$ One study reported the development of squamous cell carcinoma on the salvaged limb which eventually required amputation. The neoplastic occurrence was detected after the removal of the fixator and was attributed to the previous chronic wound status of the patient. ${ }^{14}$ One patient sustained a supracondylar fracture upon removal of the fixator, which was treated conservatively, unfortunately, the same patients sustained another fracture on the same region which was also treated non-surgically. The patient recovered and was able to proceed to full weight-bearing thereafter. ${ }^{18}$ Thirty-five percent of the patients reported residual stiffness and a degree of loss of motion described by ASAMI. Despite the reported loss of motion and other complications, the functional results were still high as previously described, and no patient deemed that amputation was a better option. ${ }^{14-17}$

This is the first systematic review on the treatment outcomes of ipsilateral medial fibular transport using the Ilizarov construct. Due to the paucity of evidence, review relied heavily on observational and non-randomized studies which are disposed to bias and random error. ${ }^{21}$ There is also a scarcity of comparison of different surgical techniques because of the lack of a standardized method of assessing results. Only the groups with measurable ASAMI 
criteria were summarized. Other interventions were either inappropriate for comparison since they belong to a different surgical population, or the reported outcomes were not well documented. Intervention studies are ideally based on randomized investigations to overcome biases, but immediate exclusion of observational studies in systematic reviews is not in conjunction with the scientific approach. Thus, leading to the conclusion that well-designed observational studies are as relevant in the decision-making regarding treatment plans as RCTs. ${ }^{22}$

\section{CONCLUSION}

The comparable external fixation time and complication rates, with a more efficient external fixation index, shows that the ipsilateral medial fibular transport using the Ilizarov frame provides a viable, less technically complicated alternate treatment option compared to microsurgical techniques for the management of substantial tibial bone defects secondary to trauma and infection.

\section{Statement of Authorship}

Both authors participated in the proposal conceptualization, data collection and analysis, and approved the final version submitted.

\section{Author Disclosure}

Both authors declared no conflicts of interest.

\section{Funding Source}

This paper was self-funded.

\section{REFERENCES}

1. Rahimnia A, Fitoussi F, Penneçot G, Mazda K. Treatment of Segmental Loss of the Tibia by Tibialisation of the Fibula: A Review of the Literature. Trauma Monthly. 2011;16(4):188-93.Puri A, Subin BS, Agarwal MG. Fibular centralisation for the reconstruction of defects of the tibial diaphysis and distal metaphysis after excision of bone tumours. The Journal of Bone and Joint Surgery British volume. 2009;91-B(2):234-9.

2. Schirò GR, Sessa S, Piccioli A, Maccauro G. Primary amputation vs limb salvage in mangled extremity: a systematic review of the current scoring system. BMC Musculoskeletal Disorders. 2015;16(1).

3. Tachdjians Pediatric Orthopaedics : John A. Herring. [Internet] [cited 2018 May 5]. Available from: https://www.bookdepository.com/ Tachdjians-Pediatric-Orthopaedics-John-Herring/9781416022213

4. MarshJL, Prokuski L, Biermann JS. Chronic Infected Tibial Nonunions With Bone Loss Conventional Techniques Versus Bone Transport. Clinical Orthopaedics and Related Research. 1994;\&NA;(301).

5. Campbell R, Berry MG, Deva A, O'Carrigan T, Harris IA. Aggressive management of tibial osteomyelitis shows good functional outcomes [Internet]. Macquarie University. Open Science Company; 1970 [cited 2018May5]. Available from: https://researchers.mq.edu.au/en/ publications/aggressive-management-of-tibial-osteomyelitis-showsgood-function
6. Parsons B, Strauss E. Surgical management of chronic osteomyelitis. The American Journal of Surgery. 2004;188(1):57-66.

7. Beris AE, Lykissas MG, Korompilias AV, Vekris MD, Mitsionis GI, Malizos KN, et al. Vascularized fibula transfer for lower limb reconstruction. Microsurgery. 2011;31(3):205-11.

8. Kinik H, Karaduman M. Cierny-Mader Type III chronic osteomyelitis: the results of patients treated with debridement, irrigation, vancomycin beads and systemic antibiotics. International Orthopaedics. 2007; 32(4):551-8.

9. Al-Sayyad M. Ipsilateral medial fibular transport using a circular external fixator for reconstruction of massive tibial bone defects in children and adolescents. The Egyptian Orthopaedic Journal. 2015;50(1):25.

10. Puri A, Subin BS, Agarwal MG. Fibular centralisation for the reconstruction of defects of the tibial diaphysis and distal metaphysis after excision of bone tumours. The Journal of Bone and Joint Surgery British volume. 2009;91-B(2):234-9.

11. Shafi R, Fragomen AT, Rozbruch SR. Ipsilateral fibular transport using Ilizarov-Taylor spatial frame for a limb salvage reconstruction: a case report [Internet]. HSS journal : the musculoskeletal journal of Hospital for Special Surgery. Springer-Verlag; 2009 [cited 2017May5]. Available from: https:/www.ncbi.nlm.nih.gov/pmc/articles/PMC2642540/

12. Paley D, Catagni MA, Argnani F, Villa A, Bijnedetti GB, Cattaneo R. Ilizarov Treatment of Tibial Nonunions With Bone Loss. Clinical Orthopaedics and Related Research. 1989;\&NA;(241).

13. Atkins, R. M., Madhavan, P., Sudhakar, J., \& Whitwell, D. Ipsilateral vascularised fibular transport for massive defects of the tibia. The Journal of Bone and Joint Surgery, 81(6), 1035-1040. 1999

14. Catagni MA, Camagni M, Combi A, Ottaviani G. Medial Fibula Transport with the Ilizarov Frame to Treat Massive Tibial Bone Loss. Clinical Orthopaedics and Related Research. 2006;448:208-16.

15. Shiha AE, Khalifa ARH, Assaghir YM, Kenawey MO. Medial transport of the fibula using the Ilizarov device for reconstruction of a massive defect of the tibia in two children. The Journal of Bone and Joint Surgery British volume. 2008;90-B(12):1627-30.

16. Yin $\mathrm{P}, \mathrm{Zhang} \mathrm{L}, \mathrm{Li}$, et al. Ipsilateral fibula transport for the treatment of massive tibial bone defects. Injury. 2015;46(11):2273-7.

17. Zaman AU, Javed S, Ahmad A, Aziz A. Massive segmental bone loss due to pantibial osteomyelitis in children reconstructed by medial fibular transport with Ilizarov frame. Journal of Taibah University Medical Sciences. 2017;12(5):418-23.

18. Kim HS, Jahng JS, Han DY, Park HW, Chun CH. Immediate ipsilateral fibular transfer in a large tibial defect using a ring fixator. International Orthopaedics. 1998Aug;22(5):321-4.

19. Chaudhary M, Jain S, Pragadeeswaran V, Lakhani P. Infected lower tibial nonunions without bone grafting - Reliable union using the Ilizarov technique. Journal of Limb Lengthening \& Reconstruction. 2015;1(1):21

20. Yin P, Ji Q, Li T, Li J, Li Z, Liu J, et al. A Systematic Review and Meta-Analysis of Ilizarov Methods in the Treatment of Infected Nonunion of Tibia and Femur. Plos One. 2015Mar;10(11).Fürmetz J, Soo C, Behrendt W, et al. Bone Transport for Limb Reconstruction Following Severe Tibial Fractures. Orthopedic Reviews. 2016;8(1): 6384. doi: 10.4081/or.2016.6384.

21. Iobst C, Liu R. A systematic review of incidence of pin track infections associated with external fixation. Journal of Limb Lengthening \& Reconstruction. 2016;2(1):6.

22. Shrier I, Boivin JF, Steele R, Platt R, Furlan A, Kakuma R, et al. Should Meta-Analyses of Interventions Include Observational Studies in Addition to Randomized Controlled Trials? A Critical Examination of Underlying Principles, American Journal of Epidemiology, 2007;(166)10:1203-9, https://doi.org/10.1093/aje/kwm189 